Article

\title{
An LCC-P Compensated Wireless Power Transfer System with a Constant Current Output and Reduced Receiver Size
}

\author{
Zhengchao Yan ${ }^{1,2}$, Yiming Zhang ${ }^{2}$, Baowei Song ${ }^{1, *}$, Kehan Zhang ${ }^{1}$, Tianze Kan ${ }^{2}$ \\ and Chris $\mathrm{Mi}^{2, *(\mathrm{D})}$ \\ 1 School of Marine Science and Technology, Northwestern Polytechnical University, Xi'an 710072, China; \\ yanzc1991@gmail.com (Z.Y.); zhangkehan210@163.com (K.Z.) \\ 2 Department of Electrical and Computer Engineering, San Diego State University, San Diego, CA 92182, USA; \\ zhangym07@gmail.com (Y.Z.); bigtreehust@gmail.com (T.K.) \\ * Correspondence: songbaowei@nwpu.edu.cn (B.S.); mi@ieee.org (C.M.); \\ Tel.: +86-29-8849-2309 (B.S.); +1-619-594-3741 (C.M.)
}

Received: 14 November 2018; Accepted: 4 January 2019; Published: 6 January 2019

\begin{abstract}
Wireless Power Transfer (WPT) for autonomous underwater vehicles (AUVs) has been a research focus in recent years. This paper studies the inductor-capacitor-capacitor and parallel (LCC-P) compensation topology to achieve a compact receiver for AUVs. Unlike the series-series (SS) compensation topology, the LCC-P topology retains the advantages of the double-sided LCC topology and has a more compact receiver than the double-sided LCC topology with fewer elements used on the receiver side. The analytical model of such a WPT system is established to analyze the output power and transfer efficiency. The LCC-P topology has a higher efficiency compared to the SS topology due to the smaller conduction loss of the inverter. Moreover, a method of eliminating the DC filter inductor $L_{0}$ is proposed to further decrease the size and weight of the receiver. The amplitude of the withstanding voltage on the receiver compensation capacitor without $L_{0}$ is approximately decreased by $40 \%$ compared to that with $L_{0}$. Both cases of with and without $L_{0}$ have a constant current output and the peak efficiency without $L_{0}$ is about $94 \%$, which is $1 \%$ lower than that with $L_{0}$. A prototype was built and the experimental results verified the theoretical analysis.
\end{abstract}

Keywords: wireless power transfer (WPT); compact receiver; constant current output; inductorcapacitor-capacitor and parallel (LCC-P)

\section{Introduction}

Wireless Power Transfer (WPT) technology is growing in popularity in recent years due to its non-physical connection between the source and the load [1-3], and it has been widely used in diverse scenarios, such as toothbrushes, cell phones [4], electrical vehicles [5], and autonomous underwater vehicles (AUVs) [6-8]. Energy is a major factor restricting the long-term continuous operation of the AUV in the ocean. Avoiding carrying too many batteries, WPT technology can be used to charge the AUV in the mission time, which can solve long-term continuous operation issue. There is an extremely restricted receiver size and weight of the receiver side on the AUV, so the receiver should be as compact as possible. The research focus covers the power electronics converters, coil optimization [9], compensation topologies [10-20], foreign object detection [21] and safety issues [22]. Among these fields, the compensation topologies, or the matching networks, are crucial to increase the overall efficiency and minimize the VA rating of the power electronics converters by using additional inductors and capacitors to adjust the reactive power. However, the additional components increase the size, weight, and cost of the system. Therefore, the amount of the additional components should be as 
few as possible, especially for the receiver side. Moreover, certain compensation topologies can be adopted to achieve a constant current output without complex control methods, which is beneficial to the battery charging.

The series-series (SS) topology is widely utilized because it has a constant current output characteristic, which is good for the charging current control. In addition, the resonant frequency is independent of the coupling coefficient and the load, which is advantageous for wireless charging [12,13]. However, the output power increases with the decreasing coupling coefficient, which will result in overcurrent under the misaligned case. Wang et al. [14] analyzed the inductor-capacitor-inductor (LCL) compensation topology for the transmitter side, where the resonant frequency is dependent of the coupling coefficient and the load. Thrimawithana et al. [15] proposed a double-sided LCL compensation topology. In this topology, open-load protection can be easily achieved and the resonant frequency is independent of the coupling coefficient and the load. However, the additional inductances are the same as or larger than those of the main coils, increasing the system cost and deteriorating the transfer efficiency. Li et al. [18] first proposed the double-sided inductor-capacitor-capacitor (LCC) compensation topology with the tuning method to achieve zero voltage switching (ZVS) for the switches of the primary inverter. The double-sided LCC compensation topology not only offers the same advantages as the double-sided LCL compensation topology, but also has the advantages of smaller additional inductances and another degree of freedom to design the system. However, in this topology, two inductors and four capacitors are introduced into the matching circuit, leading to a high cost and large size. To decrease the size, weight, and cost of the receiver side and reserve the merits of double-sided LCC compensation topology, this paper studies LCC-P compensation topology, which has the advantages of resonant frequency independent of the coupling coefficient and load, constant primary current, and constant current output. It also has the merit of fewer additional components on the receiver side, meaning a smaller size and a lower cost compared with the double-sided LCC topology.

In this paper, the WPT system with an LCC-P compensation topology is studied to make the receiver more compact. The gap distance is fixed at $60 \mathrm{~mm}$ with no misalignment, because the AUV can automatically align the transmitter and the receiver and we also have some mechanical devices to improve the docking precision. The analytical model is established to analyze the system characteristics. Circuit simulation is applied to verify the proposed method of further reducing the receiver size and weight. A prototype is built and experiments are conducted to verify the analysis.

This paper is organized as follows: the circuit analysis of the LCC-P topology with and without $L_{0}$ is presented in Section 2. The experiments of a prototype of the LCC-P topology are conducted and compared with the calculation and simulation in Section 3. The conclusions are drawn in Section 4.

\section{Circuit Analysis}

The topology and the equivalent circuit of the wireless charging system with an LCC-P compensation topology are depicted in Figure $1 . \mathrm{S}_{1}-\mathrm{S}_{4}$ are four power MOSFETs of the inverter and $\mathrm{D}_{1}-\mathrm{D}_{4}$ are the rectifier diodes. $U_{\text {in }}$ and $U_{1}$ denote the $\mathrm{DC}$ and AC voltages of the inverter, respectively. $L_{1}$ and $L_{2}$ are the self-inductances of the transmitter and the receiver. $L_{\mathrm{f}}, C_{\mathrm{f}}, C_{1}$, are the primary-side compensation inductor and capacitor and $C_{2}$ is the secondary-side compensation capacitor. $R_{L f}, R_{1}$, and $R_{2}$ represent the equivalent resistances of $L_{\mathrm{f}}, L_{1}$, and $L_{2}$, respectively. $I_{L f}, I_{C f}, I_{1}, I_{2}, I_{C 2}, I_{L}$, $U_{L}$ denote the root-mean-square (RMS) values of the corresponding variables. $L_{0}$ and $C_{0}$ are the filter inductor and the filter capacitor. $U_{\text {bat }}$ and $I_{\text {bat }}$ denote the battery voltage and the battery current, respectively. $M$ is the mutual inductance of the transmitter and the receiver.

In order to realize a constant transmitter current and the protection of the primary side when the secondary side is taken away, we use the resonant conditions of the double-sided LCC topology on the primary side [18]. $L_{\mathrm{f}}$ resonates with $C_{\mathrm{f}}$, which forms an $\mathrm{LC}$ resonant circuit. When this LC resonant circuit is fed with a voltage source, the output current of the LC resonant circuit is constant. Also, the series reactance of $L_{1}$ and $C_{1}$ resonates with $C_{\mathrm{f}}$, therefore, $L_{1}, C_{1}$, and $C_{\mathrm{f}}$ form a parallel resonant 
circuit when the secondary side is taken away, which can protect the primary side. $C_{2}$ is selected to resonate with $L_{2}$ :

$$
\omega_{0} L_{\mathrm{f}}-\frac{1}{\omega_{0} C_{\mathrm{f}}}=\omega_{0} L_{1}-\frac{1}{\omega_{0} C_{1}}-\frac{1}{\omega_{0} C_{\mathrm{f}}}=\omega_{0} L_{2}-\frac{1}{\omega_{0} C_{2}}=0
$$

where $\omega_{0}$ is the resonant angular frequency.

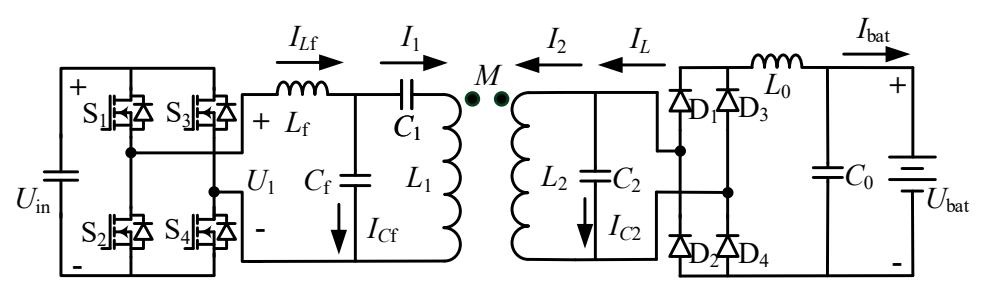

(a)

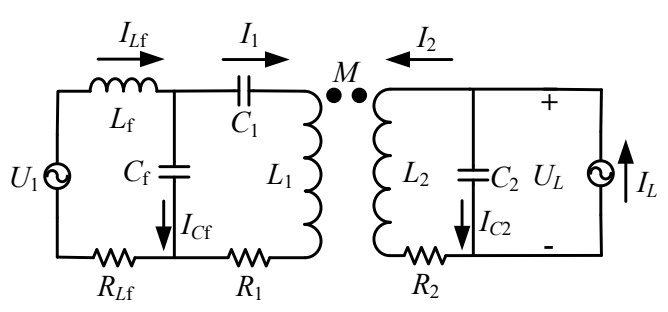

(b)

Figure 1. WPT system with LCC-P compensation (a) Topology (b) Equivalent circuit.

\subsection{Theoretical Analysis}

Based on the Kirchhoff's law, the equations of the equivalent circuit can be obtained:

$$
\left\{\begin{aligned}
\mathbf{U}_{1} & =\left(R_{L \mathrm{f}}+j \omega L_{\mathrm{f}}\right) \mathbf{I}_{L \mathrm{f}}+\frac{\mathbf{I}_{C \mathrm{f}}}{j \omega \mathrm{C}_{\mathrm{f}}} \\
& =\left(R_{L \mathrm{f}}+j \omega L_{\mathrm{f}}\right) \mathbf{I}_{L \mathrm{f}}+\left(j \omega L_{1}+\frac{1}{j \omega C_{1}}+R_{1}\right) \mathbf{I}_{1}+j \omega M \mathbf{I}_{2} \\
\mathbf{U}_{L} & =\left(j \omega L_{2}+R_{2}\right) \mathbf{I}_{2}+j \omega M \mathbf{I}_{1}=\frac{\mathbf{I}_{C 2}}{j \omega C_{2}} \\
\mathbf{I}_{L f} & =\mathbf{I}_{C \mathrm{ff}}+\mathbf{I}_{1}, \mathbf{I}_{L}=\mathbf{I}_{2}+\mathbf{I}_{C 2}
\end{aligned}\right.
$$

where $\omega$ is the operating angular frequency. The variables in bold denote their phasor quantities. Without considering the equivalent resistances of the compensation inductor, the transmitter, and the receiver and at the resonant state, the transmitter current, the $\mathrm{AC}$ current of the rectifier, and $\mathrm{AC}$ current of the inverter are:

$$
\mathbf{I}_{1}=\frac{\mathbf{U}_{1}}{j \omega_{0} L_{\mathrm{f}}}, \mathbf{I}_{L}=-\frac{M \mathbf{U}_{1}}{j \omega_{0} L_{\mathrm{f}} L_{2}}, \mathbf{I}_{L \mathrm{f}}=-\frac{M \mathbf{I}_{2}}{L_{\mathrm{f}}}
$$

It can be seen that the AC current of the rectifier is independent of the load, which indicates that the proposed system has a constant current output. The battery current keeps constant due to the large filter inductor $L_{0}$ so that the AC current of the rectifier is a square wave. With the Fourier Decomposition, $U_{1}$ and $I_{L}$ can be obtained as [23]:

$$
U_{1}=\frac{2 \sqrt{2}}{\pi} U_{\text {in }}, I_{L}=\frac{2 \sqrt{2}}{\pi} I_{\text {bat }}
$$

Assume that the power on both sides of the rectifier is identical so that the AC voltage of the rectifier can be expressed as:

$$
U_{L}=\frac{\pi}{2 \sqrt{2}} U_{\text {bat }}
$$


However, the phase differences of the currents and voltages are unknown. Based on the defined directions in Figure 1b, the phasor diagram of all the AC currents and voltages is plotted in Figure 2.

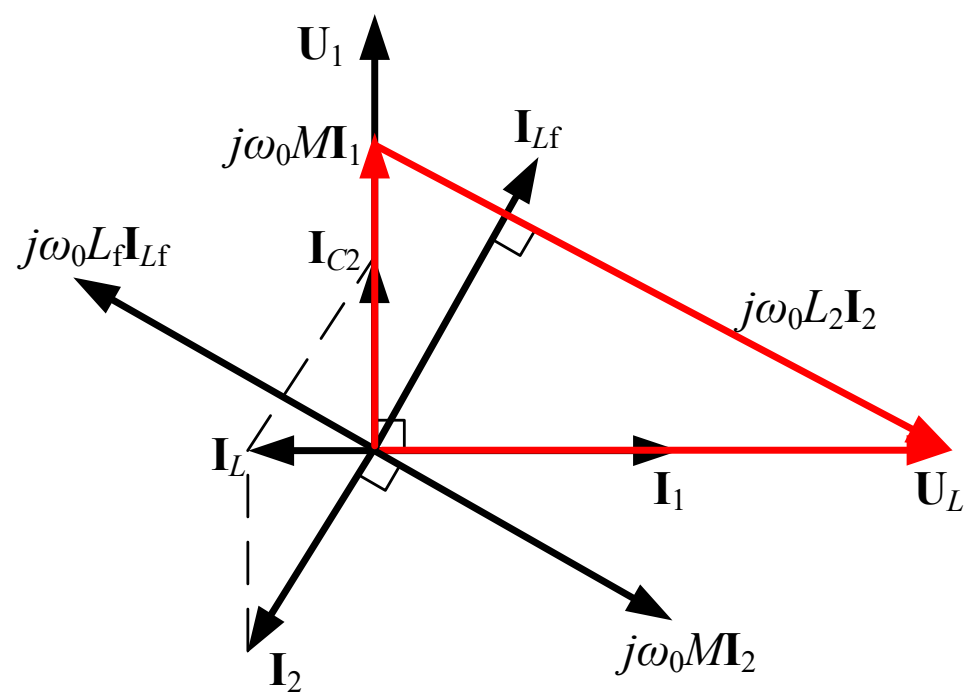

Figure 2. Phasor diagram of a WPT system with the LCC-P topology.

It can be seen that the input voltage leads the input current, which indicates that the ZVS is achieved inherently. Based on the Pythagorean theorem in the red triangle, the receiver current can be calculated as:

$$
I_{2}=\frac{\sqrt{U_{L}^{2}+\left(\omega_{0} M I_{1}\right)^{2}}}{\omega_{0} L_{2}}=\frac{U_{\text {in }}}{\omega_{0}} \sqrt{\frac{\pi^{2}}{8 L_{2}^{2}} G^{2}+\frac{8 k^{2}}{\pi^{2} \alpha^{2} L_{1} L_{2}}}
$$

where $G, k$, and $\alpha$ denote the voltage gain, coupling coefficient, and ratio of the compensation inductance and the primary self-inductance, respectively, which can be expressed as:

$$
G=\frac{U_{\text {bat }}}{U_{\text {in }}} ; k=\frac{M}{\sqrt{L_{1} L_{2}}} ; \alpha=\frac{L_{\mathrm{f}}}{L_{1}}
$$

Substituting (6) into (3), the AC current of the inverter can be obtained:

$$
I_{L \mathrm{f}}=\frac{k U_{\text {in }}}{\alpha \omega_{0}} \sqrt{\frac{\pi^{2}}{8 L_{1} L_{2}} G^{2}+\frac{8 k^{2}}{\pi^{2} \alpha^{2} L_{1}^{2}}}
$$

It can be seen that the $\mathrm{AC}$ current of the inverter $I_{L \mathrm{f}}$ decreases with the decreasing coupling coefficient $k$. Therefore, when the receiver is misaligned or taken away from the transmitter, the system circuit can be protected from overcurrent. The ratio of $I_{L f}$ and $I_{1}$ is defined as:

$$
r=\frac{I_{L f}}{I_{1}}=\sqrt{\frac{\pi^{4} k^{2} L_{1}}{64 L_{2}} G^{2}+\frac{k^{4}}{\alpha^{2}}}
$$

We choose $L_{1}=L_{2}$ for simplicity. Figure 3 shows the ratio varying with the coupling coefficient $k$ under different $\alpha$ or under different $G$. In most of practical applications of a loosely coupled WPT system, $k$ is no larger than 0.5 . It can be seen that the ratio is no more than 1 when $k$ is below 0.5 , which indicates that the large current does not flow through the MOSFETs of the inverter compared with the SS compensation topology, leading to a smaller conduction loss of the inverter. 


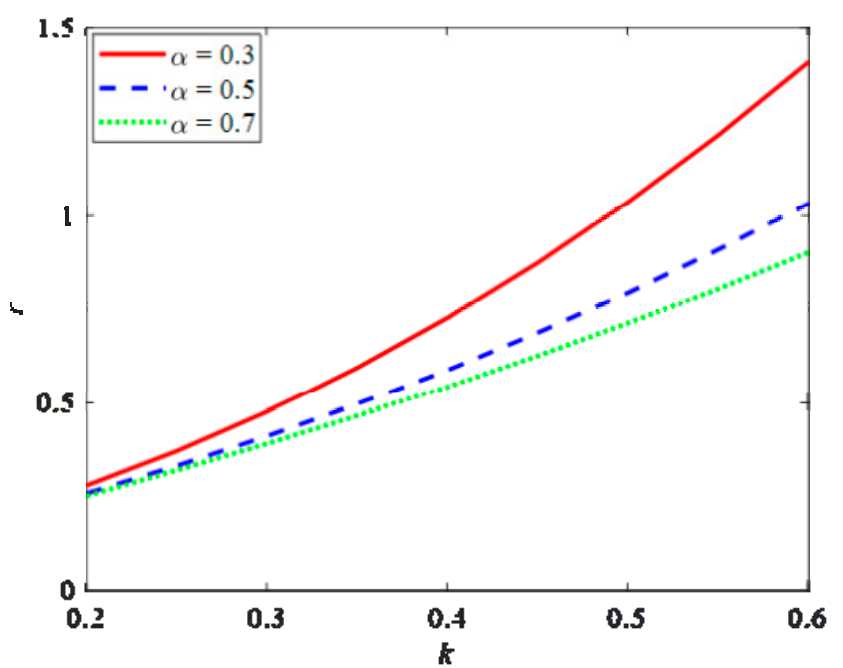

(a)

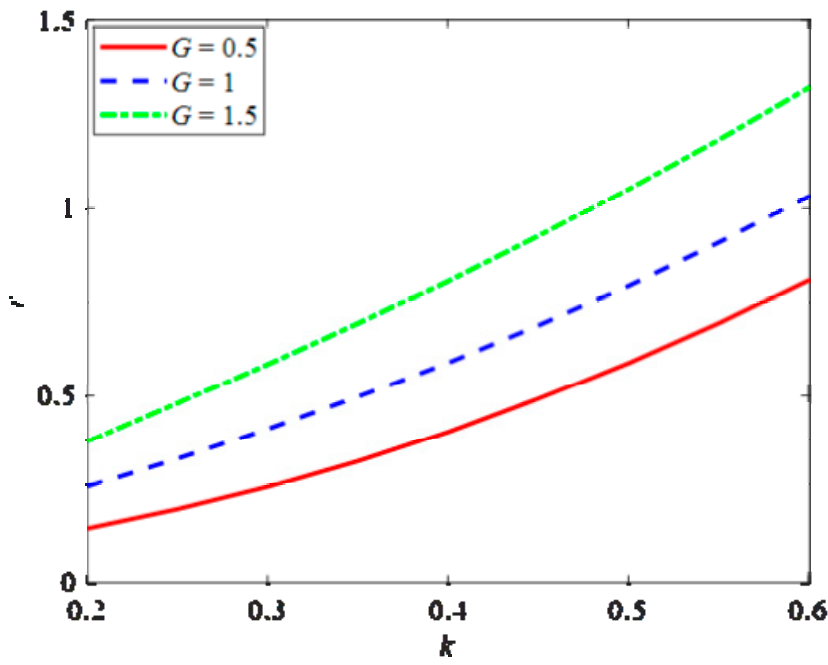

(b)

Figure 3. The ratio of $I_{L f}$ and $I_{1}$ varying with $k(\mathbf{a})$ Under different $\alpha(\mathbf{b})$ Under different $G$.

The output power can be calculated as:

$$
P_{\text {out }}=U_{L} I_{L}=\frac{M U_{\text {in }} U_{\text {bat }}}{\omega_{0} \alpha L_{1} L_{2}}
$$

It can be noted that the output power is proportional to the input voltage $U_{\text {in }}$, the battery voltage $U_{\text {bat }}$, and the mutual inductance $M$. Assuming that $R_{1}=R_{2}$ and $L_{1}=L_{2}$ in the system, the transfer efficiency $\eta$ can be expressed as:

$$
\begin{aligned}
\eta & =\frac{P_{\text {out }}}{P_{\text {out }}+I_{1}^{2} R_{1}+I_{2}^{2} R_{2}+I_{L f}^{2} R_{L f}} \\
& 1+\frac{\pi^{2}}{8}\left(\frac{\alpha}{k Q}+\frac{k}{Q_{\mathrm{f}}}\right) G+\frac{8}{\pi^{2}}\left(\frac{1}{k \alpha Q}+\frac{k}{\alpha Q}+\frac{k^{3}}{\alpha^{2} Q_{\mathrm{f}}}\right) \frac{1}{G}
\end{aligned}
$$

where $Q$ is the quality factor of the transmitter and the receiver, and $Q_{\mathrm{f}}$ is the quality factor of the compensation inductor, defined by:

$$
Q=\frac{\omega_{0} L_{1}}{R_{1}}=\frac{\omega_{0} L_{2}}{R_{2}}, Q_{\mathrm{f}}=\frac{\omega_{0} L_{\mathrm{f}}}{R_{L \mathrm{f}}}
$$


It can be obviously seen that there is a maximum transfer efficiency with respect to the voltage gain $G$. The transfer efficiency is maximized when:

$$
G=\frac{8}{\pi^{2}} \sqrt{\frac{\alpha Q_{\mathrm{f}}+k^{2} \alpha Q_{\mathrm{f}}+k^{4} Q}{\alpha^{3} Q_{\mathrm{f}}+k^{2} \alpha^{2} Q}}
$$

The maximum transmission efficiency is:

$$
\eta_{\max }=\frac{1}{1+2 \sqrt{\frac{1+k^{2}}{k^{2} Q^{2}}+\frac{1+2 k^{2}}{\alpha Q Q_{\mathrm{f}}}+\frac{k^{4}}{\alpha^{2} Q_{\mathrm{f}}^{2}}}}
$$

Figure 4 shows the maximum efficiency varying with $\alpha$, which is the ratio of $L_{\mathrm{f}}$ and $L_{1}$. It indicates that the maximum efficiency increases with the increasing of $\alpha$ and then becomes a relatively stable value under different coupling coefficient $k$. Therefore, $\alpha$ can be set to 0.5 to have a relatively high efficiency under different coupling and avoid too large compensation inductance.

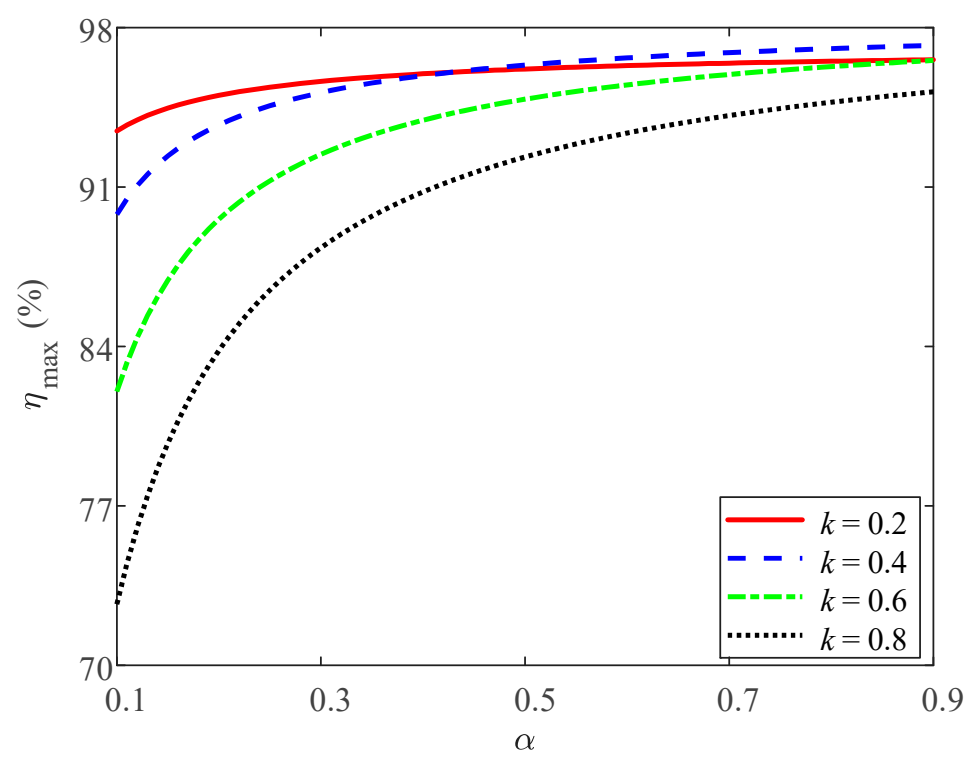

Figure 4. The maximum efficiency varying with $\alpha$.

\subsection{Eliminating $L_{0}$}

In the practical application, the receiver size and weight should be minimized, because the space on the AUV is limited to install the device. To further decrease the receiver volume, the large filter inductor $L_{0}$ can be eliminated, as depicted in Figure $5 \mathrm{a}$. It can be noted that the compensation capacitor $C_{2}$ before the rectifier is parallel to the filter capacitor $C_{0}$, leading to the fact that the diode bridge comes into conduction only when the voltage on $C_{2}$ exceeds the battery voltage. As a result, the voltage on $C_{2}$ is clamped to the battery voltage for the large filter capacitor. Therefore, the withstanding voltage of $C_{2}$ can be reduced significantly. Thus, the size, weight, and cost of the receiver are further decreased for practical applications. Meantime, the inductance of the leading wire $L_{\mathrm{w}}$, depicted in dashed line in Figure 5a, should be considered under this case. The waveforms of $U_{1}, U_{L}$, and $i_{L}$ are shown in Figure $5 \mathrm{~b}$. Only the first half cycle is analyzed due to the half-wave symmetrical waveforms. $t_{1}$ is the point where the voltage on $C_{2}$ exceeds the battery voltage, $t_{2}$ is the point where the inverter switches, and $T$ is the switching period. 


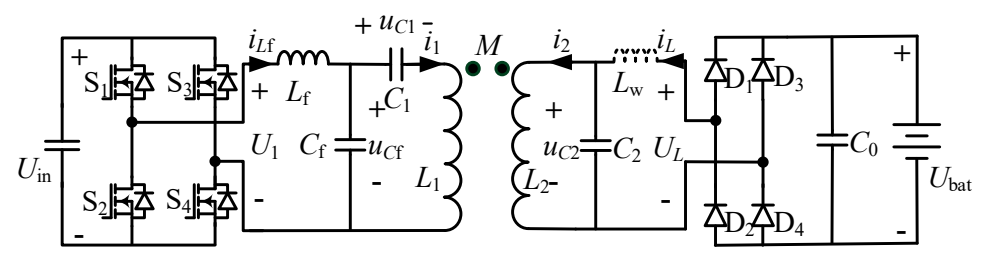

(a)

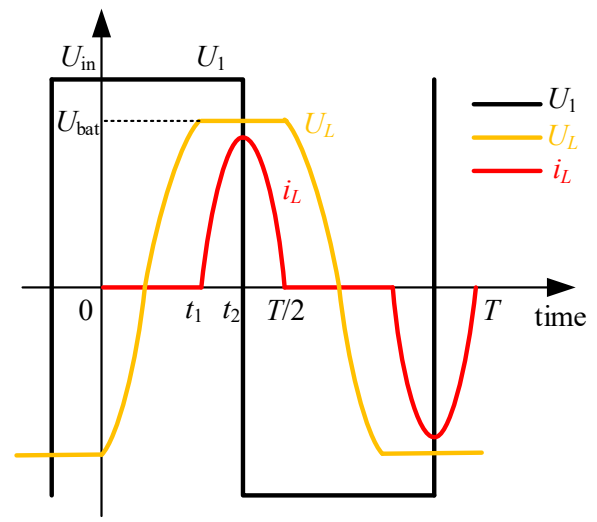

(b)

Figure 5. The topology and the waveforms (a) Topology without the large filter inductor (b) Waveforms of the inverter and the rectifier.

Take the inductor currents and the capacitor voltages $i_{1}, i_{2}, i_{L f}, i_{L}, u_{C 1}, u_{C 2}$, and $u_{C f}$ as the state variables, the WPT system with the LCC-P compensation topology has three working modes, which are defined as Mode 1, 2, and 3.

Mode $1\left[0-t_{1}\right]$ : In this mode the rectifier is not conducted until $t_{1} \cdot i_{L}$ equals zero and $u_{C 2}$ equals $U_{L}$. The model in Figure 5 for Mode 1 can be established by:

$$
\left\{\begin{array}{l}
U_{\text {in }}=L_{\mathrm{f}} \frac{d i_{L f}}{d t}+u_{C f}, \\
u_{C f}=u_{C 1}+L_{1} \frac{d i_{1}}{d t}+M \frac{d i_{2}}{d t}, u_{C 2}=L_{2} \frac{d i_{2}}{d t}+M \frac{d i_{1}}{d t} \\
i_{L f}=C_{f} \frac{d u_{C f}}{d t}+i_{1}, C_{2} \frac{d u_{C 2}}{d t}+i_{2}=0 \\
i_{1}=C_{1} \frac{d u_{C 1}}{d t}
\end{array}\right.
$$

Mode $2\left[t_{1}-t_{2}\right]$ : In this mode the rectifier is conducted with $S_{1}, S_{4}, D_{1}$, and $D_{4}$ on. $U_{L}$ equals $U_{\text {bat }}$. This mode ends at $t_{2}$ where the inverter switches. The model in Figure 5 for Mode 2 can be established by:

$$
\left\{\begin{array}{l}
U_{\mathrm{in}}=L_{\mathrm{f}} \frac{d i_{\mathrm{Lf}}}{d t}+u_{\mathrm{Cf}}, U_{\mathrm{bat}}=L_{\mathrm{W}} \frac{d i_{L}}{d t}+u_{\mathrm{C} 2} \\
u_{\mathrm{Cf}}=u_{\mathrm{C} 1}+L_{1} \frac{d i_{1}}{d t}+M \frac{d i_{2}}{d t}, u_{\mathrm{C} 2}=L_{2} \frac{d i_{2}}{d t}+M \frac{d i_{1}}{d t} \\
i_{L f}=C_{\mathrm{f}} \frac{d u_{\mathrm{Cf}}}{d t}+i_{1}, i_{\mathrm{L}}=C_{2} \frac{d u_{C 2}}{d t}+i_{2} \\
i_{1}=C_{1} \frac{d u_{C 1}}{d t}
\end{array}\right.
$$

Mode $3\left[t_{2}-T / 2\right]$ : In this mode the rectifier is conducted with $S_{2}, S_{3}, D_{1}$, and $D_{4}$ on. $U_{L}$ also equals $U_{\text {bat }}$ and this mode ends at $T / 2$ where $i_{L}$ reaches zero. The model in Figure 5 for Mode 3 can be established by:

$$
\left\{\begin{array}{l}
-U_{\text {in }}=L_{\mathrm{f}} \frac{d i_{L f}}{d t}+u_{\mathrm{Cf}}, U_{\mathrm{bat}}=L_{\mathrm{w}} \frac{d i_{L}}{d t}+u_{\mathrm{C} 2} \\
u_{\mathrm{Cf}}=u_{C 1}+L_{1} \frac{d i_{1}}{d t}+M \frac{d i_{2}}{d t}, u_{\mathrm{C} 2}=L_{2} \frac{d i_{2}}{d t}+M \frac{d i_{1}}{d t} \\
i_{L f}=C_{\mathrm{f}} \frac{d u_{C f}}{d t}+i_{1}, i_{L}=C_{2} \frac{d u_{C 2}}{d t}+i_{2} \\
i_{1}=C_{1} \frac{d u_{C 1}}{d t}
\end{array}\right.
$$


Combining the initial conditions, the differential equations can be solved. However, it is too complicated to get the closed-form expression. Therefore, the circuit simulation method is adopted which is introduced in Section 3.

\section{Calculations, Simulations, and Experiments}

In order to verify the aforementioned theoretical analysis, an experimental prototype is implemented, as shown in Figure 6. The system specifications and the circuit parameters are listed in Table 1 . The parameters of the transmitter and the receiver are approximately equal.

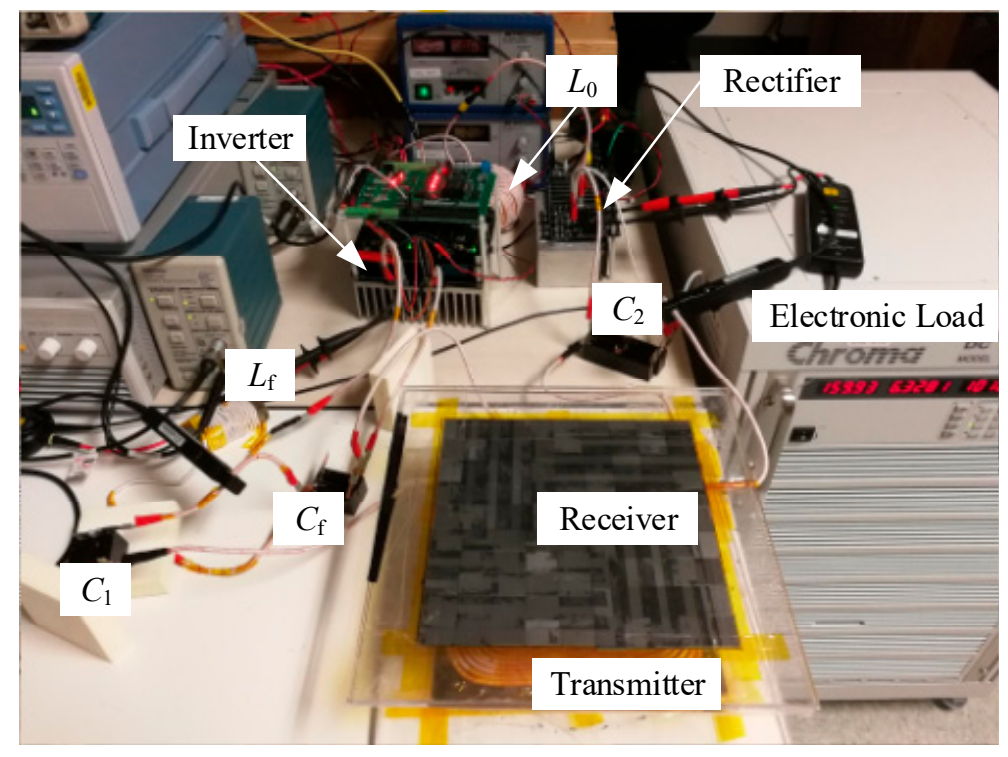

Figure 6. Experimental prototype.

Table 1. System Specifications and Circuit Parameters.

\begin{tabular}{ccc}
\hline Note & Symbol & Value \\
\hline Transmitter inductance & $L_{1}$ & $49.57 \mu \mathrm{H}$ \\
Receiver inductance & $L_{2}$ & $49.05 \mu \mathrm{H}$ \\
Compensation inductance & $L_{\mathrm{f}}$ & $24.8 \mu \mathrm{H}$ \\
Transmitter-side parallel compensation capacitance & $C_{\mathrm{f}}$ & $153.4 \mathrm{nF}$ \\
Transmitter-side series compensation capacitance & $C_{1}$ & $153.4 \mathrm{nF}$ \\
Transmitter-side series compensation capacitance & $C_{2}$ & $77.6 \mathrm{nF}$ \\
Filter inductance & $L_{0}$ & $713 \mu \mathrm{H}$ \\
Coupling coefficient & $k$ & 0.36 \\
Number of turns & $N_{1}, N_{2}$ & 12 \\
Gap & $d$ & $60 \mathrm{~mm}$ \\
Coil dimension & & $200 \mathrm{~mm} \times 200 \mathrm{~mm}$ \\
Resonant frequency & $f_{0}$ & $81.6 \mathrm{kHz}$ \\
\hline
\end{tabular}

A full-bridge inverter is employed at the transmitter side with Silicon Carbide Power MOSFET C2M0080120D as the switches. A full-bridge rectifier is utilized at the receiver side with Fast Recovery Epitaxial Diode DSEI120-06A as the switches. The transmitter and the receiver coils are composed of tightly wound AWG $38 \mathrm{Litz}$ wires with a $3.9 \mathrm{~mm}$ diameter. The ratio of the compensation inductance and the transmitter inductance $\alpha$ is set to 0.5 . The inverter input voltage, the inverter input current, the battery voltage, the battery current, the input power, the output power, and the DC-DC efficiency are all measured by a YOKOGAWA WT1800 power analyzer. The filter inductor $L_{0}$ is $713 \mu \mathrm{H}$, which is large enough to make the rectifier current stable. 


\subsection{Comparison of LCC-P and SS Topology}

Compared to the double-sided LCC compensation topology, the proposed WPT system with the LCC-P topology can reduce the receiver size and weight, which indicates a lower cost and a higher efficiency. Moreover, the SS topology has the same amount of compensation components on the secondary side and a constant current output, so it is necessary to make a comparison of these two topologies. Figure 7 shows the circuit topology with the SS compensation. $C_{3}\left(C_{4}\right)$ is the compensation capacitance of the transmitter (receiver) side. The only difference of the circuit topology with the LCC-P compensation is the values of the compensation capacitances. In the SS compensation, the operating frequency is the same as the LCC-P compensation as Table 1 shows. $C_{3}=76.7 \mathrm{nF}, C_{4}=77.6 \mathrm{nF}, U_{\text {in }}$ is fixed at $100 \mathrm{~V}$ and $U_{\text {bat }}$ is changing. For the LCC-P compensation, the circuit parameters are set as Table $1 . U_{\text {in }}$ is fixed at $160 \mathrm{~V}$ and $U_{\text {bat }}$ is changing.

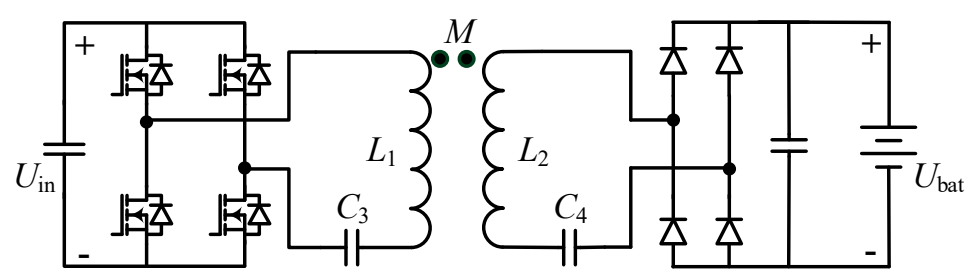

Figure 7. The circuit topology with SS compensation.

The DC-DC efficiency of the WPT system with LCC-P and SS compensation topologies versus output power is shown in Figure 8.

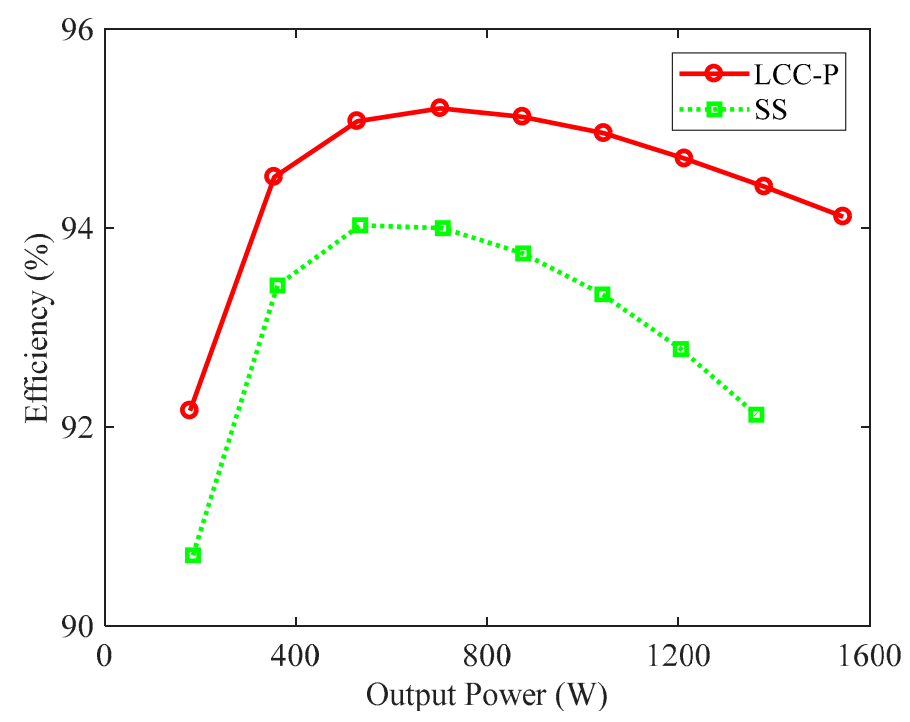

Figure 8. The system efficiency varying with the output power.

It can be seen that the efficiency of LCC-P compensation topology is larger than the SS compensation topology. The peak efficiency of the LCC-P topology is $1 \%$ larger than the SS compensation topology. It is because SiC MOSFETs (C2M0080120D), the drain-source on-state resistance of which are typically $80 \mathrm{~m} \Omega$, are used in the inverter and smaller input current flow through the inverter, leading to lower conduction loss of the inverter as Section 2.1 analyzed. Moreover, the output power of the SS topology increases with the decreasing mutual inductance, that is to say, the system circuit could be overcurrent when misalignments arise. Nevertheless, this defect can be eliminated by using LCC-P compensation topology, the output power of which decreases with the decrease of mutual inductance. Therefore, the LCC-P compensation topology is the optimal selection through the above comparison. 


\subsection{LCC-P Compensation Topology}

In order to investigate the relationship of the DC-DC efficiency and the voltage gain, the output power is maintained the same because the efficiency of the power electronics components changes with the varying output power. The gap distance is $60 \mathrm{~mm}$ with no misalignment. By regulating the output power to be $1000 \mathrm{~W}$ and changing the voltage gain, the DC-DC efficiency varying with the voltage gain is plotted in Figure 9. It indicates that the efficiency has a sharp increase with the voltage gain and peaks at $95 \%$, and then declines moderately. The experimental results well match the calculated ones. We choose the maximum DC-DC efficiency point as the measurement point of the losses of different parts, where $U_{\text {in }}=183 \mathrm{~V}, U_{\text {bat }}=200 \mathrm{~V}$.

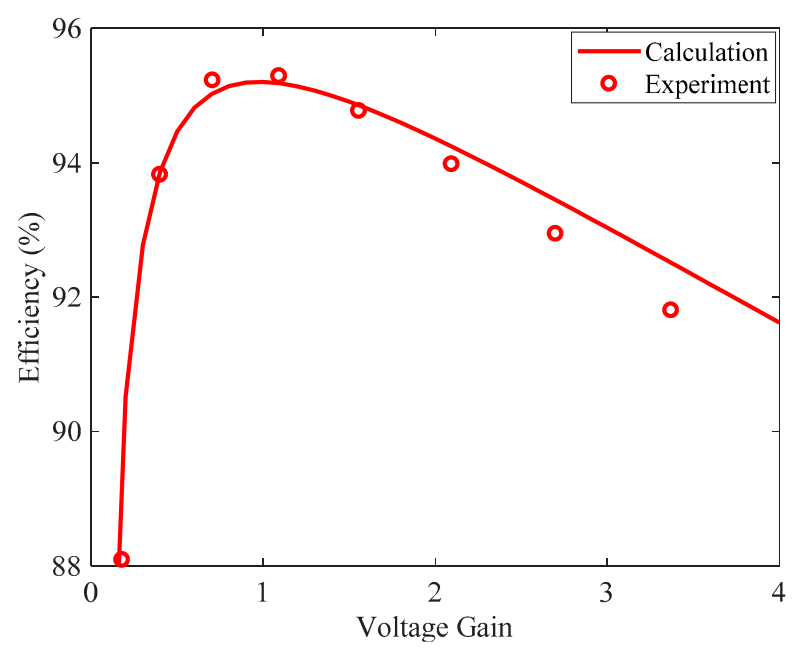

Figure 9. System efficiency varying with the voltage gain.

Figure 10 shows the power losses distributions at this point. It can be seen that most losses localize in the inverter and the transmission coils. By regulating the input voltage to be $160 \mathrm{~V}$ and changing the battery voltage, the DC-DC efficiency and output power varying with the battery voltage is depicted in Figure 11. In the experiment, the input voltage is fixed at $160 \mathrm{~V}$ by a DC power source and an electronic load with a constant voltage is used to represent the battery for adjusting the voltage. It can be seen that the efficiency has a rapid increase with the rising battery voltage and peaks at a certain battery voltage and then drops slowly. The maximum efficiency is about $95 \%$. The output power is proportional to the battery voltage, which indicates that the battery current is independent of the load. Therefore, it is easily controlled to charge the battery. The calculation results are verified by the experimental results.

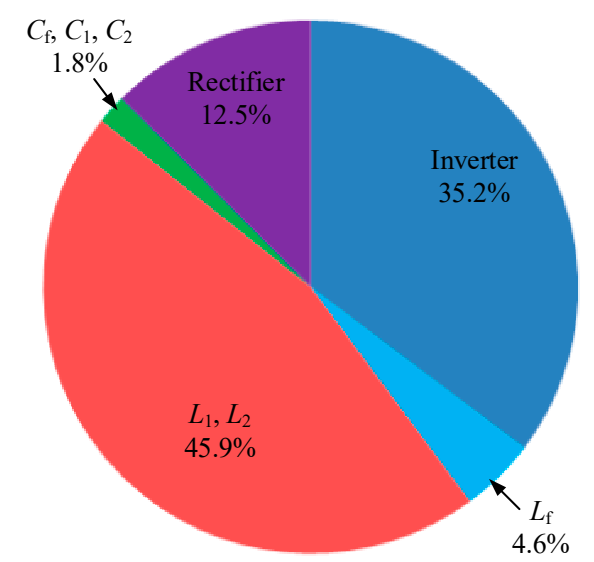

Figure 10. Power losses distributions when $U_{\text {in }}=183 \mathrm{~V}, U_{\text {bat }}=200 \mathrm{~V}$. 


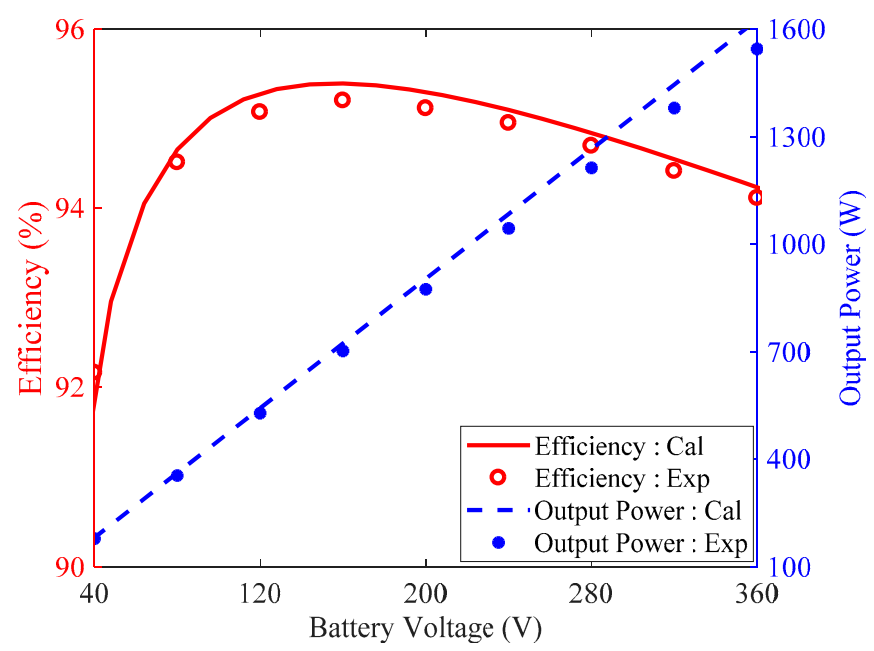

Figure 11. The efficiency and output power varying with the battery voltage.

\subsection{Eliminating $L_{0}$}

The size and weight of the receiver side are extremely restricted the AUVs. The simulation software Matlab Simulink (2017a) is utilized to study the characteristics of the proposed method in Section 2.2. The parameters are set in Table 1. The inductance of the wire is $0.7 \mu \mathrm{H}$. When $U_{\text {in }}=160 \mathrm{~V}$, the simulated and experimental waveforms of the AC voltage and current of the rectifier with and without $L_{0}$ are shown in Figure 12. It can be seen that the AC voltage of the rectifier is squared off, which indicates that the voltage on $C_{2}$ is clamped to the battery voltage. The amplitude of the voltage on $C_{2}$ without $L_{0}$ is roughly decreased by $40 \%$ compared with that with $L_{0}$. Thus, the receiver size and weight are further decreased for practical applications.
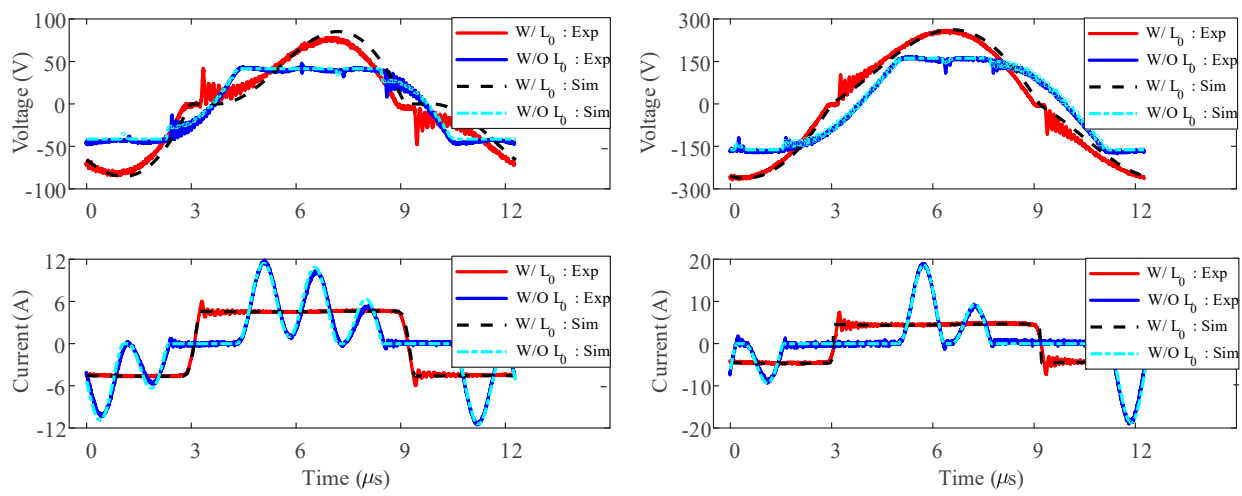

(b)
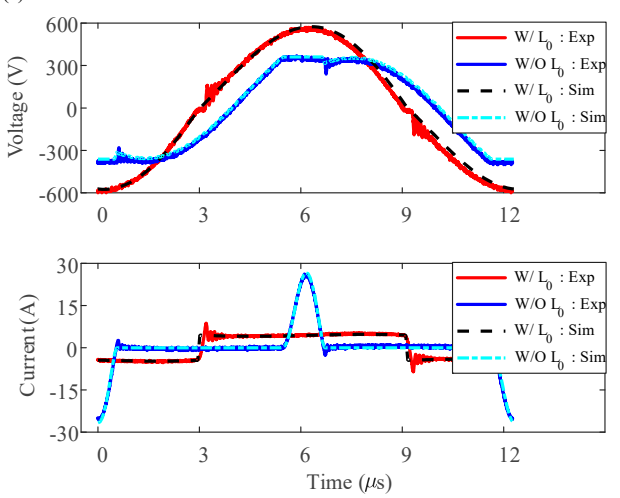

(c)

Figure 12. AC voltage and current waveforms of the rectifier (a) $U_{\text {bat }}=40 \mathrm{~V}$ (b) $U_{\text {bat }}=160 \mathrm{~V}$ (c) $U_{\text {bat }}=360 \mathrm{~V}$. 
The AC current of the rectifier without $L_{0}$ is discontinuous and has much higher peaks and oscillations in this case. With the increasing battery voltage, the amount of the pulses decrease from three to one due to the voltage amplitudes of the ripples on $C_{2}$ are more difficult to exceed the battery voltage. The experimental results match the simulated results well.

The simulated and experimental battery currents varying with the battery voltage with and without $L_{0}$ are shown in Figure 13. It can be seen that the battery current keeps constant with the increasing battery voltage, which indicates that the WPT system with LCC-P compensation topology has a constant current output under both cases. The experimental results agree well with the simulated ones.

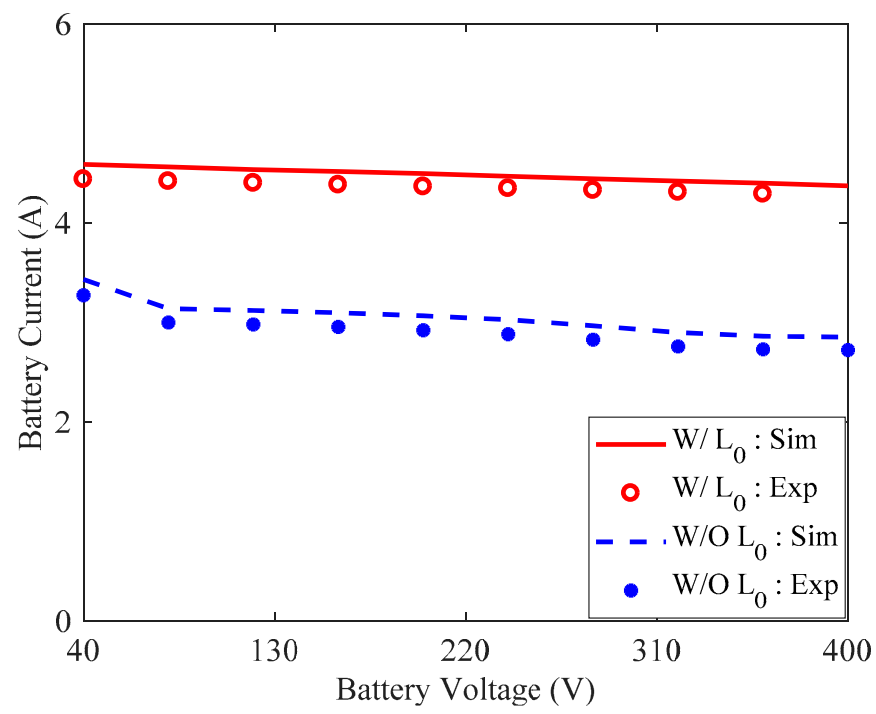

Figure 13. The battery current with the battery voltage.

The output power and the DC-DC efficiency varying with the battery voltage are plotted in Figure 14. The input voltage $U_{\text {in }}$ is fixed at $160 \mathrm{~V}$. It indicates that the output power is proportional to the battery voltage under both cases. However, the power level without $L_{0}$ is lower than that with $L_{0}$. The experimental DC-DC efficiencies of both cases are maximized at a certain battery voltage. The peak efficiency without $L_{0}$ is about $94 \%$, which is only $1 \%$ lower than that with $L_{0}$.

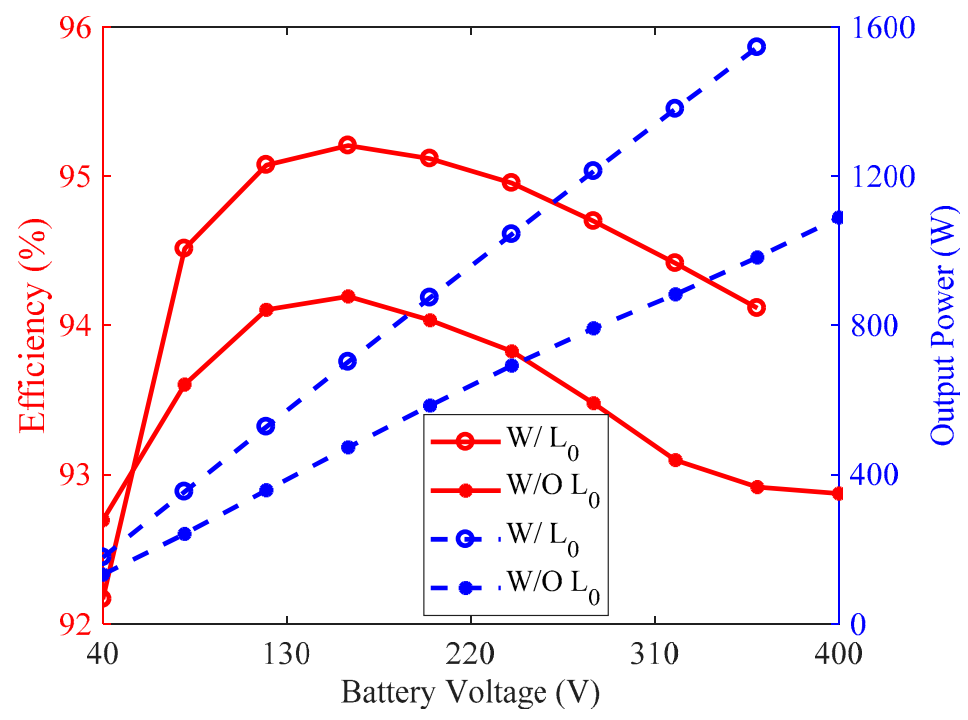

Figure 14. The output power and DC-DC efficiency varying with the battery voltage. 
Although the output power and the efficiency are slightly affected by eliminating the large filter inductor $L_{0}$, the withstanding voltage on $C_{2}$ is reduced significantly and the large DC inductor is eliminated. Therefore, the LCC-P compensation topology without the filtering inductor $L_{0}$ can be used in some applications where there is a restricted receiver side space.

\section{Conclusions}

In this paper, the WPT system with an LCC-P compensation topology has been modeled and analyzed. The proposed compensation topology has a more compact receiver side. The expressions of the output power and transfer efficiency have been derived. The output power and the transfer efficiency have been analyzed for different voltage gains. Moreover, the DC filter inductor can be eliminated to further decrease the receiver size and weight. A prototype has been implemented and the experimental results have verified the theoretical analysis.

It is found that the DC-DC efficiency of the WPT system with an LCC-P compensation topology is larger than that with the SS topology due to a lower conduction loss of the inverter. The output power is proportional to the battery voltage and the transfer efficiency can be maximized with the voltage gain. Moreover, the withstanding voltage on $C_{2}$ is clamped to the battery voltage. The amplitude of the voltage on $C_{2}$ without $L_{0}$ is roughly decreased by $40 \%$ compared to the case with $L_{0}$. Both cases with and without $L_{0}$ have a constant current output, which can be easily controlled to charge the battery. The peak efficiency without $L_{0}$ is about $94 \%$, which is only $1 \%$ lower than that with $L_{0}$. Therefore, it is practicable to the decrease the receiver size and weight by eliminating the filter inductor.

Author Contributions: Z.Y. handled the technical modeling and drafted the manuscript. Z.Y., Y.Z. and T.K. designed the experiments and analyzed the data. B.S. and K.Z. contributed to the circuit design and revised the manuscript. C.M. contributed the topology design, experiment and revised the manuscript.

Funding: This research was funded by the Natural Science Basic Research Plan in Shaanxi Province of China (Grant No. 2018JM5033) and the China Scholarship Council (Grant No. 201706290074).

Acknowledgments: The authors would like to acknowledge the financial support from the Natural Science Basic Research Plan in Shaanxi Province of China (Grant No. 2018JM5033) and the China Scholarship Council (Grant No. 201706290074).

Conflicts of Interest: The authors declare no conflict of interest.

\section{References}

1. Li, S.Q.; Mi, C.C. Wireless Power Transfer for Electric Vehicle Applications. IEEE J. Emerg. Sel. Top. Power Electron. 2015, 3, 4-17.

2. Zhang, Y.M.; Lu, T.; Zhao, Z.M.; He, F.B.; Chen, K.N.; Yuan, L.Q. Employing Load Coils for Multiple Loads of Resonant Wireless Power Transfer. IEEE Trans. Power Electron. 2015, 30, 6174-6181. [CrossRef]

3. Liu, X.; Wang, T.F.; Yang, X.J.; Tang, H.J. Analysis of efficiency improvement in wireless power transfer system. IET Power Electron. 2018, 11, 302-309. [CrossRef]

4. Momeneh, A.; Castilla, M.; Ghahderijani, M.M.; Miret, J.; de Vicuna, L.G. Analysis, design and implementation of a residential inductive contactless energy transfer system with multiple mobile clamps. IET Power Electron. 2017, 10, 875-883. [CrossRef]

5. Buja, G.; Rim, C.T.; Mi, C.T.C. Dynamic Charging of Electric Vehicles by Wireless Power Transfer. IEEE Trans. Ind. Electron. 2016, 63, 6530-6532. [CrossRef]

6. Kan, T.; Mai, R.; Mercier, P.P.; Mi, C. Design and Analysis of a Three-Phase Wireless Charging System for Lightweight Autonomous Underwater Vehicles. IEEE Trans. Power Electron. 2018, 33, 6622-6632. [CrossRef]

7. Yan, Z.; Zhang, K.; Wen, H.; Song, B. Research on characteristics of contactless power transmission device for autonomous underwater vehicle. In Proceedings of the OCEANS 2016, Shanghai, China, 10-13 April 2016.

8. Zhang, K.H.; Yan, L.B.; Yan, Z.C.; Wen, H.B.; Song, B.W. Modeling and analysis of eddy-current loss of underwater contact-less power transmission system based on magnetic coupled resonance. Acta Phys. Sin. 2016, 65, 048401-1.

9. Kim, S.; Covic, G.A.; Boys, J.T. Comparison of Tripolar and Circular Pads for IPT Charging Systems. IEEE Trans. Power Electron. 2018, 33, 6093-6103. [CrossRef] 
10. Villa, J.L.; Sallan, J.; Osorio, J.F.S.; Llombart, A. High-Misalignment Tolerant Compensation Topology For ICPT Systems. IEEE Trans. Ind. Electron. 2012, 59, 945-951. [CrossRef]

11. Zhang, W.; Mi, C.C. Compensation Topologies of High-Power Wireless Power Transfer Systems. IEEE Trans. Veh. Technol. 2016, 65, 4768-4778. [CrossRef]

12. Zhang, Y. Key Technologies of Magnetically-Coupled Resonant Wireless Power Transfer; Springer: Singapore, 2017.

13. Valtchev, S.; Borges, B.; Brandisky, K.; Klaassens, J.B. Resonant Contactless Energy Transfer With Improved Efficiency. IEEE Trans. Power Electron. 2009, 24, 685-699. [CrossRef]

14. Wang, C.S.; Covic, G.A.; Stielau, O.H. Investigating an LCL load resonant inverter for inductive power transfer applications. IEEE Trans. Power Electron. 2004, 19, 995-1002. [CrossRef]

15. Thrimawithana, D.J.; Madawala, U.K. A Generalized Steady-State Model for Bidirectional IPT Systems. IEEE Trans. Power Electron. 2013, 28, 4681-4689. [CrossRef]

16. Hou, J.; Chen, Q.H.; Wong, S.C.; Tse, C.K.; Ruan, X.B. Analysis and Control of Series/Series-Parallel Compensated Resonant Converter for Contactless Power Transfer. IEEE J. Emerg. Sel. Top. Power Electron. 2015, 3, 124-136.

17. Su, Y.; Tang, C.; Wu, S.; Sun, Y. Research of LCL resonant inverter in wireless power transfer system. In Proceedings of the International Conference on Power System Technology, PowerCon 2006, Chongqing, China, 22-26 October 2006.

18. Li, S.Q.; Li, W.H.; Deng, J.J.; Nguyen, T.D.; Mi, C.C. A Double-Sided LCC Compensation Network and Its Tuning Method for Wireless Power Transfer. IEEE Trans. Veh. Technol. 2015, 64, 2261-2273. [CrossRef]

19. Li, W.H.; Zhao, H.; Deng, J.J.; Li, S.Q.; Mi, C.C. Comparison Study on SS and Double-Sided LCC Compensation Topologies for EV/PHEV Wireless Chargers. IEEE Trans. Veh. Technol. 2016, 65, 4429-4439. [CrossRef]

20. Wang, Y.J.; Yao, Y.S.; Liu, X.S.; Xu, D.G. S/CLC Compensation Topology Analysis and Circular Coil Design for Wireless Power Transfer. IEEE Trans. Transp. Electr. 2017, 3, 496-507. [CrossRef]

21. Jeong, S.Y.; Kwak, H.G.; Jang, G.C.; Choi, S.Y.; Rim, C.T. Dual-purpose Non-overlapping Coil Sets as Metal Object and Vehicle Position Detections for Wireless Stationary EV Chargers. IEEE Trans. Power Electron. 2018, 33, 7387-7397. [CrossRef]

22. Zhang, K.H.; Du, L.N.; Zhu, Z.B.; Song, B.W.; Xu, D.M. A Normalization Method of Delimiting the Electromagnetic Hazard Region of a Wireless Power Transfer System. IEEE Trans. Electromagn. C 2018, 60, 829-839. [CrossRef]

23. Zhang, Y.; Zhao, Z.; Jiang, Y. Modeling and analysis of wireless power transfer system with constant-voltage source and constant-current load. In Proceedings of the 2017 IEEE Energy Conversion Congress and Exposition (ECCE), Cincinnati, OH, USA, 1-5 October 2017. 